\title{
Stratégies de vie sur une frontière. Le cas du Maramures : enjeux et valorisations identitaires
}

Life strategies on a border and the case of the Maramureş region: the stakes and valorisation of identity

Estrategias de vida en la frontera: el caso de Maramureş. Implicaciones y

valorizaciones identitarias

\section{Raluca NAGY}

\section{CpenEdition}

Journals

Édition électronique

URL : http://journals.openedition.org/conflits/17329

DOI : $10.4000 /$ conflits. 17329

ISSN : $1777-5345$

Éditeur :

CCLS - Centre d'études sur les conflits lilberté et sécurité, L'Harmattan

Édition imprimée

Date de publication : 20 décembre 2008

Pagination : 45-55

ISBN : 1157-966X

ISSN : 1157-996X

Référence électronique

Raluca NAGY, "Stratégies de vie sur une frontière. Le cas du Maramures : enjeux et valorisations identitaires », Cultures \& Conflits [En ligne], 72 | hiver 2008, mis en ligne le 19 mai 2009, consulté le 30 mars 2021. URL : http://journals.openedition.org/conflits/17329 ; DOI : https://doi.org/10.4000/ conflits. 17329 


\title{
Stratégies de vie sur une frontière. Le cas du Maramures : enjeux et valorisations identitaires
}

\author{
Raluca NAGY
}

Raluca Nagy est doctorante en sciences sociales à l'université libre de Bruxelles et à l'Ecole nationale de sciences politiques et administratives de Bucarest.

\section{Introduction géo-historique à la région de Maramures}

$\mathrm{D}$ u fait de sa position géographique et de sa réalité historique, le Maramures est depuis toujours une région assez isolée, bénéficiant d'une autonomie interne importante.

L'Etat-nation roumain est né de l'union de la Moldavie et de la Valachie en 1859. A l'issue de la Première Guerre mondiale, la Transylvanie, qui appartenait à l'Empire austro-hongrois, s'est vue rattachée à la Roumanie. Le Maramures est, quant à lui, l'une des sous-régions de la Transylvanie. Les deux autres grandes provinces historiques roumaines, la Moldavie et la Valachie, forment ce que l'on appelle le « Regat ». Ce rapprochement tardif de la Transylvanie va jouer un rôle important dans le rapport périphérie-centre qui va s'instituer entre la Transylvanie et le « Regat» 1 .

Lors de l'occupation romaine de la Dacie (équivalent de la Roumanie actuelle) le Maramures a su rester le territoire dit des «Daces libres », contrairement au reste de la Transylvanie. Comment s'est alors opérée la romanisation des Maramureseni ? L'hypothèse selon laquelle ceux-ci ont été romanisés par d'autres Daces romanisés antérieurement pourrait se justifier. L'un des mythes fondateurs du Maramures va en effet dans ce sens.

1. Après la Seconde Guerre mondiale, la Roumanie perd plusieurs parties de ses territoires, comme par exemple la Bucovine du Nord, la Bessarabie - c'est à dire une partie de ce qui est aujourd'hui la Moldavie - et le Sud du Dobrudja. Elle conserve cette «forme » jusqu’à nos jours 
Sous le règne de Vladislav (Laslau), les Tartares envahirent la Transylvanie. Vladislav se rendit à Rome pour demander de l'aide et l'Empereur accepta de lui envoyer tous les bandits qui croupissaient dans ses prisons, à une seule condition : une fois leur tâche accomplie, il ne devait pas les renvoyer à Rome. Vladislav les marqua en leur rasant les cheveux et les emmena faire la guerre à ses côtés. Les Tartares furent ainsi chassés de l'autre coté du Siret, la rivière qui servait de frontière. Les Hongrois, croyant que ces bandits allaient se retrouver sur leurs terres, les « installèrent » au Maramures. C'est ainsi que les bandits se virent récompensés d'avoir lutté aux côtés des hommes de Vladislav, avec un territoire et des terres bien à eux ${ }^{2}$.

Le Maramures fut placé sous un contrôle hongrois que l'on peut qualifier de « léger » à partir du XIe siècle, tout en restant une région autonome jusqu'à son inclusion dans le Royaume hongrois, au XIVe siècle. Officiellement placée sous diverses occupations et marquée par une instabilité continue, la province historique du Maramures (différente du département du même nom qui existe actuellement en Roumanie) fut rattachée à la Tchécoslovaquie en 1919, pour être ensuite reprise en totalité par la Hongrie entre 1940 et 1944, puis intégrée, depuis 1945, à l'Ukraine (soviétique à ce moment-là) ${ }^{3}$. Dans le Sud du Maramures historique, la majorité de la population est roumaine. S'y ajoutent trois minorités : hongroise, ukrainienne (certains auteurs considèrent que ce sont des Ruthènes, pour d'autres, il s'agit de Houtsouls) et rom. Dans le Nord, la majorité est ukrainienne ; néanmoins, de petites communautés roumaines et hongroises coexistent. Le Maramures dont il est question dans cet article constitue seulement la partie roumaine du Maramures historique qui se retrouve dans le département roumain portant le même nom.

De l'époque du «territoire des Daces libres » jusqu'à sa « division » entre la Roumanie et l'Ukraine, le Maramures garde donc une certaine autonomie, parfois plus officieuse qu'officielle, due à sa position stratégique et frontalière, mais aussi à sa distance par rapport aux capitales (capitales successives des pays auxquels il a appartenu).

Cette autonomie ne l'empêche pourtant pas, en tant que zone frontalière, d'attirer des populations diverses qui sont passées dans cette région et qui s'y sont établies, mélangées ou qui parfois ont presque entièrement disparu (voir l'histoire des juifs de Maramures).

Cette dualité entre la «pureté dace » d'un côté, et le mélange de populations et de communautés de l'autre, est un jeu de fermeture et d'ouverture caractéristique de toutes les régions frontalières. Cette situation est très bien

2. Mesnil M., Popova A., «Etranger de tout poil ou comment on désigne l'autre », Civilisations, n०42, 1993, pp. 179-198.

3. Karnoouh C., L'Invention du peuple, Paris, Editions Arcantère, 1990, p. 18. 
illustrée par la métaphore utilisée par le sociologue roumain H.H. Stahl. Ce dernier traite de la particularité des zones de frontière et du rôle qu'elles jouent dans le cadre des régions élargies en décrivant ce qu'il nomme l'«effet de culasse 4 ». Cette image montre la pression culturelle que la position de frontière exerce sur la mobilisation plus aiguë des traditions comme forme de justification de l'authenticité et, finalement, de résistance culturelle. Pour illustrer cet effet de culasse, Stahl donne justement l'exemple de la zone frontalière commune à la Hongrie, la Slovaquie, la Ruthénie (l'Ukraine d'aujourd'hui) : le Maramures. Le Maramures historique a été notamment colonisé à partir de 1728 par des juifs exilés de Russie, puis en 1746 par les habitants de la ville slovaque de Tipz. L'hypothèse de Stahl est l'idée selon laquelle que tous ces changements et mouvements de population dus à sa position frontalière n'ont fait que renforcer la tradition de la population majoritaire les Roumains - et mettre en avant leur "pureté » et le fait que les habitants soient des descendants directs, non métissés, des Daces.

Géographiquement, cette authenticité préservée pourrait s'expliquer par son statut de «zone ethnographique archä̈que 5 » ou par ce qu'on appelle en géographie humaine un « isolat », c'est-à-dire une zone rendue difficilement accessible par sa position dans une région montagneuse, éloignée de toute grande route commerciale. Ce type de zone est donc moins touché par les diverses influences extérieures. Des régions comme le Maramures, Oas, Campulung, Vrancea, Mehedinti, Padureni ou Tara Motilor ont toutes en commun cette « authenticité » préservée.

\section{Discours sur l'authenticité}

Bénéficiant de ces caractéristiques d'authenticité, le Maramures est une des régions ayant parfaitement servi aux discours de la construction de l'Etat national fondé sur le "paysan parfait ». Cette image bucolique a bien été intégrée dans un discours idéologique sur la paysannerie roumaine, avec ses valeurs et ses pratiques atemporelles.

L'adjectif « authentique » renvoie à un objet ou un phénomène qui correspond à un original, un réel. Exigeant des preuves (pedigree, patine), l'authenticité est fragile ${ }^{6}$. Les anthropologues évitent généralement l'usage de ce

4. La culasse est soit la pièce en métal qui sert à fermer les cylindres d'un moteur en propageant la dynamique et la puissance, soit la partie d'une arme qui a les mêmes effets. On trouve ce concept dans le rapport de S. Messmann et L. Conley Barrows présenté au Symposium international « Les relations interrégionales dans le domaine de l'éducation, de la science, la culture, et la communication en Europe centrale et du Sud-Est », Bucarest, 19-22 avril 2001.

5 . Ianosi I., Octavian G., Patroescu M., Rey V., Atlasul Romaniei, Bucarest, Editura Enciclopedia Rao, 2006.

6. Heinich N., «Authentique ? », Terrain, n³3, septembre 1999, http://terrain.revues.org/document2673.html, mis en ligne le 11 juillet 2005. 
qualificatif à cause, justement, de son ambivalence et de son renvoi permanent à une dichotomie «vrai » / «faux». Ils utilisent par conséquent un vocabulaire plus vague, avec des termes comme «symbolique », «significatif », «pertinent », etc. Il existe un contraste fort entre ces usages et les visions des personnes concernées qui utilisent elles-mêmes le terme de «tradition » avec toutes les connotations d'authenticité et de véridicité qu'il sous-entend pour se comparer à d'autres personnes. Les habitants de Maramures perçoivent l'authenticité à différents degrés attachés aux sous-régions ${ }^{7}$. C'est ainsi que les habitants de la Vallée de Viseu se disent plus «modernes», mais moins «authentiques » que ceux de la Vallée d'Iza : "Ici nous n'avons pas de traditions comme ceux de la Vallée d'Iza, nous les avons malheureusement perdues, mais c'est aussi parce qu'on a été plus en contact avec le monde avec le train [chemin de fer existant dans la vallée] et tout ». Les habitants de la Vallée d'Iza, eux, se sentent moins «traditionnels » que ceux de Gutai, et ainsi de suite.

Mais le dilemme mis en discussion par le discours anthropologique sur l'authenticité nous mène à deux types d'approches pour l'invention culturelle en anthropologie 8 :

- ceux qui font la différence entre "authentique » et "inventé », entre «vrai » et «faux ». Par exemple, Hobsbawm tient un discours sur la tradition réelle (genuine) et la tradition inventée (spurious), en soutenant que l'invention apparait particulièrement dans des situations de changement social rapide ou de discontinuités flagrantes avec le passé; la thèse de Hobsbawm est très importante pour cette étude de cas, dans la mesure où le contexte présente, entre autres, les caractéristiques qu'il évoque 9.

- ceux qui soutiennent que la tradition est un processus, donc modifiable et modifié par défaut et qui trouvent que « tradition inventée » serait un pléonasme. Par exemple, Linnekin estime que la tradition est un processus, une interprétation continue du passé, qu'elle est donc toujours réinventée, chan-

7. Comme nous l'avons déjà mentionné, ce texte a une approche principalement anthropologique (en respectant les théories de l'anthropologie économique et de l'économie informelle ainsi que celles qui concernent les mobilités) et se fonde sur une recherche doctorale intitulée « La marchandisation des traditions. Etude de cas sur un groupe de villages dans le Maramures, Roumanie ». Notre recherche de terrain s'est déroulée entre 2003 et 2006 dans trois sousrégions du Maramures historique : des villages situés autour du mont Gutai, dans la Vallée d'Iza et dans la Vallée de Viseu. Ces cinq enquêtes de terrain ont duré environ un mois chacune, couvrant toutes les saisons de l'année pour avoir un aperçu aussi complet que possible des phénomènes touristique et migratoire, à travers l'observation participante et non participante. Ce texte accorde une attention particulière aux villages ukrainiens de la troisième sousrégion, la Vallée de Viseu, notamment Ruscova.

8. Linnekin J., "Cultural Invention and the Dilemma of Authenticity", American Anthropologist, n'93, 1991, pp. 446-449.

9. Hobsbawm E., Ranger T. (eds.), The Invention of Tradition, New York, Cambridge University Press, 1992. 
gée. «Vrai » ou «faux » deviennent des termes inappropriés quand on les applique à la tradition, un phénomène social qui n'existe pas en dehors de l'interprétation qu'on lui donne.

Les anthropologues post-modernes (notamment Clifford, Marcus et Fisher) ont par ailleurs changé radicalement la façon de définir la culture authentique. C'est aussi pour cette raison que, depuis ces dernières années, le poids du discours sur l'authenticité tend à s'amoindrir en anthropologie.

Pour revenir à notre objet, on assiste à un discours invariable sur l'authenticité du Maramures, discours repris pendant les années de communisme, avec un grand nombre de contradictions et de dissimulations : pour promouvoir «l'homme nouveau », l'ouvrier, on avait besoin du paysan. Les villages de cette région tranquille et traditionnelle ont continué à vivre comme ils le faisaient depuis des siècles, mais pas seulement pour des raisons idéologiques : l'économie, et surtout l'agriculture, y ont également joué un rôle prépondérant.

\section{Pratiques, stratégies, « débrouille »}

L'une des raisons économiques pour lesquelles cette région, tout comme d'autres zones ethnographiques archaïques, a été « préservée » durant le communisme repose sur le manque d'intérêt et/ou l'hostilité dont elle témoignait vis-à-vis de la collectivisation ${ }^{10}$, à cause de la pauvreté de son sol ou de la grande distance la séparant de la capitale. Le paysage rural, une fois encore, n'a donc pas été atteint par les influences extérieures.

Derrière le discours d'authenticité et de pureté rurale de la région, la réalité s'avère néanmoins plus nuancée. Lorsqu'on parle de l'ouverture et de la flexibilité impliquées par cette localisation frontalière spécifique, ce n'est pas uniquement dans un sens culturel, mais aussi dans un sens économique. Afin de mieux comprendre les stratégies de vie développées par les habitants du Maramures, qui reprennent parfois même ce discours d'authenticité et l'utilisent à leurs propres fins économiques, il paraît utile d'aborder certaines notions.

Tandis que l'image archétypale du paysan roumain est toujours présente, celui-ci est confronté à une réalité assez dure, à une agriculture qui dérape et qui n'arrive pas à le soutenir, au choc prolongé de la période dite « de transition », entre socialisme et capitalisme, et de l'entrée du pays dans l'Union

10. Kideckel D.A., The Solitude of Collectivism: Romanian Villagers to the Revolution and Beyond, Ithaca / London, Cornell University Press, 1993. Dans son analyse de la collectivisation et ses effets après 1990, David Kideckel suggère que le système socialiste a échoué dans sa réalisation de la collectivisation, c'est-à-dire la mise en commun des propriétés foncières dans de grandes fermes (CAP : Coopérative agricole de production) appartenant à l'Etat. Cette collectivisation a finalement eu l'effet contraire, nuisant aux valeurs et arrangements collectifs. 
européenne, avec des règles de marché qui viennent rendre encore plus difficile la situation des paysans. Il faut aussi saisir la complexité de ces paysans qui ne vivent, pour la plupart, plus exclusivement de l'agriculture. Les régions archaïques et montagneuses comme le Maramures n'ont été collectivisées que partiellement et ses habitants n'ont jamais uniquement vécu, même bien avant le communisme, de l'agriculture ou des activités liées. Au Maramures, comme partout en Roumanie, les paysans (sur)vivent donc en diversifiant leurs activités et stratégies économiques. Kearney utilise le terme "polybian 11 » («détenteur de plusieurs vies»), pour rendre compte de cette habileté à manier plusieurs identités économiques. Derrière cette image de paysan parfait, il y a donc en vérité le "paysan à mi-temps 12 » dont parle Mesnil ou le «paysan-ouvrier 13 » évoqué par Carrier. Etant donné que les ruraux sont pris de plus en plus dans la sphère des biens et de la marchandisation - concepts qui ont longtemps dominé les sciences sociales, comme le traditionalisme ou le retard économique -, la production de subsistance ou la misère ont été rejetés. La vision des années 1950 et 1960 des paysans comme culturellement incapables de répondre au changement a été remplacée par celle d'une réelle capacité de flexibilité et d'adaptabilité face au changement.

Depuis toujours pour les uns, ou plus récemment, après les années 1990 pour les autres, les paysans ont donc été inscrits dans une formule économique complexe. Des auteurs comme Diminescu ou Mihailescu parlent de «pluriactivité 14 » ou de "pluriactivité complexe 15 », c'est-à-dire d'une «multistratégie » de vie, une composition d'opportunités que les habitants ont très rapidement saisie et dont ils ont profité, ou dont ils continuent à se servir chacun à leur façon. Cette "débromille 16 ", souvent envisagée seulement comme une opportunité pour sortir d'une situation difficile, constitue une possibilité d'amélioration du niveau de vie par l'acquis d'un «surplus », un ensemble de stratégies multiples d'adaptation mouvante à un système qui, lui même, évolue. La multiplicité des formes composant ce bricolage et la variété des tactiques qui lui donnent naissance sont le résultat des comportements et des motivations des acteurs de ce système. La question qui se pose ici est de savoir si ce phénomène apparaît comme lié au nouveau contexte économique ou s'il fait écho à des pratiques et modes d'être-au-monde plus anciens.

11. Kearney M., Reconceptualising the Peasantry: Anthropology in Global Perspective, Bulder, Colo. Westview, 1996, pp. 141

12 . M. Mesnil, en parlant de l'activité minière assez importante dans quelques sous-régions du Maramures qui n'est pourtant jamais mentionnée pour ne pas nuire au discours de pureté.

13. Carrier J.G. (ed.), A Handbook of Economic Anthropology, Cheltenham UK, Edward Elgar Publishing, 2005.

14 . Diminescu D., Lagrave R., « Pour une anthropologie des migrations roumaines en France. Le cas du Pays d'Oas ", Migrations Etudes, n91, novembre-décembre 1999, p. 5.

15. Mihailescu V., "What is Anthropology at Home Good for in Times of Transition?", http://www.ssn.msh-paris.fr/gb/paper\%20Mihalescu.html

16 . Lagneaux S., Nagy R., Negre R., "Between Communism and Capitalism, Resourcefulness in Everyday Life", à paraître dans Revue d'Histoire, Bucarest, 2008. 
Cette manière d'affronter le présent constitue un fil d'Ariane entre les diverses périodes historiques roumaines, notamment dans les pratiques de survie développées sous le communisme. Confrontés aux changements post1990, les gens se tournent donc davantage vers une poursuite de la «débrouille » héritée, tout en l'ancrant dans la «nouveauté » du système aujourd'hui âgé de 20 ans. En effet les populations roumaines ont toujours développé une adaptation face aux diverses situations de domination et formes de pouvoir. Ces expériences inscrites dans la longue durée semblent avoir été reconduites dans la nouvelle situation provoquée par l'ouverture du marché et des frontières. L'immobilisme du paysan roumain n'en serait alors pas un. S'il existe bien une permanence et une autochtonie, elle n'est pas aussi massive que les représentations occidentales ou nationales roumaines du paysan ne le laissent entendre.

Après 1990, c'est le tourisme qui a repris au nationalisme la construction d'un discours sur la tradition et l'authenticité.

\begin{abstract}
« Nulle part en Europe (ni ailleurs) la tradition paysanne n'a pu résister au déploiement du monde urbain, industriel, mercantile et socialiste ; mais, ce qui me parait caractériser l'Europe orientale c'est qu'avant même qu'il ne lui donne assaut, le mouvement nationaliste lui avait déjà préparé le terrain dans l'espace culturel ${ }^{17}$. »
\end{abstract}

Ce modèle « villageois » hors du temps, totalement idéalisé, se prête parfaitement à la promotion touristique; les touristes veulent voir une des sociétés qui constituent les derniers signes visibles de l'état archaïque du monde. En échange, une telle société répond aux attentes et aux demandes d'authenticité par une véritable mise en scène des traditions. Nous avons un premier exemple de stratégie de vie ou de «débrouille» que les Maramuresenis, avec une histoire du tourisme qui date des années 1970 18, ont vite compris. Mais, afin de mieux expliciter cette continuité de la «pluriactivité » dans un contexte frontalier, il convient de nous concentrer sur le cas de la migration de travail.

17. Karnoouh C., op. cit., p. 63.

18. L'intérêt pour la région s'est d'abord limité à celui des chercheurs qui faisaient une collecte ethnographique d'objets qui s'intéressaient au folklore de la région,. Jusqu'à la fin des années 1960, la région n’est presque pas touchée par le tourisme. Les chercheurs étrangers arrivent à partir de la fin des années 1960 et du début des années 1970, d'abord les «Européens » et les «francophones » (M. Mesnil en 1968, C. Karnooh en 1971) et, à partir des années 1975, les chercheurs américains (G. Kligman). Cette arrivée de "scientifiques » étrangers au Maramures se voit doublée du développement du tourisme interne, généré par le courant et la mode « ethno » des années 1960-1970 (les albums photographiques, les meubles archaïques, etc.) 


\section{La migration de travail : enjeux et valorisations identitaires sur une frontière}

La migration pendulaire est un phénomène relativement récent qui a visiblement explosé dans les dix dernières années.

«L'effondrement des régimes communistes dans les pays d'Europe centrale et orientale a conduit, entre autres phénomènes, à donner une visibilité à des mouvements de circulation migratoire différents de ceux d'avant 1989 qui étaient, pour leur part, ancrés dans une longue tradition d'émigration sans possibilité de retour. Les migrations définitives sont devenues rares, tandis que le va-et-vient est bien plus important qu'avant ${ }^{19}$. »

Au Maramures, la migration de travail semble pérenne. Le nomadisme actuel qui touche le Maramures comme d'autres zones à la périphérie de l'Europe s'inscrit dans une certaine tradition :

«Les raisons du nomadisme actuel s'inscrivent dans une longue histoire de recherche du travail, puisque les conditions des terres [...] ne permettent pas, et n'ont jamais permis, de vivre de l'agriculture. La mobilité est, en effet, un fait structurel de cette région, produit d'une longue habitude historique. L'élevage ovin et le travail forestier ont été deux occupations principales et traditionnelles de cette population. [...] Ainsi, les paysans ont-ils été contraints à la pluriactivité (complexe) qui, en l'absence d'autres secteurs d'activités [...], les a conduits à une mobilité interne à la région, puis au pays tout entier ${ }^{20}$. »

Une deuxième pratique, tout aussi importante, est l'habitude de passer la frontière et de se déplacer dans les pays voisins, avec toutes les activités informelles que cela implique. Commerce illicite d'or sur la rivière qui sert de frontière avec l'Ukraine ou migration interne sous le communisme, autant de pratiques qui forment les pionniers de la migration «à l'Ouest» après les années 1990. La mobilité demeure en effet un fait structurel de cette région frontalière, due en grande partie à la position géographique, mais aussi à l'adaptabilité et la caractéristique bien ancrée de pluriactivité des paysans. Si l'improvisation économique dans laquelle vivent ces gens de frontière a des racines et une continuité historique, il est également intéressant de se pencher sur les motivations, les représentations et les changements en cours.

19. Diminescu D., Lagrave R., op. cit., p. 2.

20. Diminescu D., Installation dans la mobilité, http://www.namediffusion.net/txtdana/mobilite_titre.html, p. 6. 
Pour reprendre les propos d'Appadurai ${ }^{21}$, la transition vers une nouvelle identité régionale est liée à un contexte de globalisation. Exposés au tourisme, les habitants de Maramures répondent au discours de berceau d'authenticité - qui est un discours valorisant venant de l'extérieur - avec la transformation de leur propre image : «si l'on vient nous voir, c'est que nous sommes dignes d'intérêt ».

Les catégorisations identitaires s'opèrent toujours en fonction d'un placement de soi par rapport à ce qui est considéré comme mieux ou pire. Ce genre de schéma peut servir d'explication et de justification des relations sociales ${ }^{22}$. Selon leurs propres dires, les habitants de Maramures se considèrent comme les «meilleurs» des Roumains. En revanche, il semblerait que les perceptions intra-régionales soient différentes.

La minorité ukrainienne ${ }^{23}$ présente dans les villages de la frontière du Maramures est presque invisible ou fait l'objet d'une discrimination négative, contrairement, par exemple, à la discrimination et aux stéréotypes positifs dont les Hongrois sont l'objet. Bien qu'ils aient une image parfois négative des Roumains ${ }^{24}$, les Ukrainiens les acceptent et les respectent, même s'ils se sentent, eux, discriminés négativement, de façon comparable voire pire que les Roms.

Il est intéressant de voir comment ces stéréotypes se sont développés et diversifiés dans la région selon les changements de contexte politique, social et économique. Il convient d'explorer la façon dont l'identité régionale et la représentation des personnes est renégociée en fonction de la « réussite migratoire ».

Pour revenir aux Ukrainiens, si avant 1990, ils constituaient une minorité souvent considérée comme plus pauvre que la majorité roumaine et peu valorisée par cette dernière, on remarque, avec la migration de travail à l'étranger, un changement net de représentation. En partant travailler à l'étranger, les Ukrainiens se «débrouillent» parfois mieux que les Roumains, gagnent davantage d'argent et finissent par « coloniser » les villages roumains, en quête

21. Appadurai A., Apres le colonialisme. Les conséquences culturelles de la globalisation, Paris, Editions Payot-Rivages, 2005.

22. Tajfel H., Human Groups and Social Categories, Cambridge, Cambridge University Press, 1981.

23. Les origines de cette minorité sont encore floues. Certains historiens pensent que cette minorité ukrainienne est composée de Ruthènes et d'autres y voient des Houtsouls («butuli», nom dérivé du roumain " hotul», voleur. Les Houtsouls forment un groupe ethnoculturel d'Ukrainiens habitant depuis des siècles les Carpates, en Ukraine, au Nord de la Roumanie (Maramures et Bucovine), mais aussi en Tchéquie, en Slovaquie et en Pologne. D’autres auteurs pensent qu'ils desendent d'une branche de slaves de l'Est, leur physionomie et leur langue étant proches de celles des slaves nordiques. Apparemment, à la frontière nord-ouest $\mathrm{du}$ Maramures, s'est établie une population venue de Lituanie (voir Visovan S., Interferente romano-ucraineene in toponimia Maramuresului, Ed. Umbria, Baia-Mare, 2001). Enfin, selon d'autres historiens encore, les bu'uli sont une population dace qui n'a pas été romanisée (les «daces libres» dont nous avons parlé précédemment). Mais, dans la mesure où ils sont tout de même proches de l'autre population slavophone habitant les Carpates, composée notamment de Ruthènes, quelques auteurs, dont Karhoouh, les appellent des « Ruthènes ». 
de terre pour bâtir leurs nouvelles maisons. Les jeunes Ukrainiens ne semblant pas fuir la campagne autant que les Roumains, les villages ukrainiens se sont vite remplis, au point que ce sont désormais les Roumains qui commencent à se sentir en minorité dans les villages roumains.

La compétence linguistique peut également constituer un exemple du basculement de statut de cette minorité. En effet, les Ukrainiens n'ont jamais été «handicapés» localement de ce point de vue et ont préféré envoyer leurs enfants dans des écoles de langue roumaine (ce qui n'est pas le cas des Hongrois par exemple). Leur motivation est aisément compréhensible et rationnelle, puisque les enfants allaient être exposés, une fois adultes, à un contexte, mais surtout un marché du travail, roumain. Cependant, l'idée plus traditionnelle selon laquelle la langue ukrainienne est peu utile en dehors du village ou du trafic transfrontalier a évolué avec les nouvelles migrations vers des contrées plus éloignées. Beaucoup d'Ukrainiens ont ainsi découvert une nouvelle utilité de leur culture et de leur langue par le biais de la reconstruction des espaces et des repères ${ }^{25}$. Leur expertise linguistique et culturelle à l'étranger est ainsi plus riche que celle des Roumains. En travaillant souvent dans la construction ou dans d'autres métiers physiques, ils se retrouvent dans des équipes de travail avec des Polonais, des Serbes ou des personnes d'autres nationalités qui parlent des langues slaves. Dans ces situations, la compréhension de l'ukrainien est un réel avantage pour des personnes qui, au départ, ne parlent pas la langue du pays d'accueil ou de migration. Dans le même temps, le fait d'avoir appris le Roumain leur offre une compétence similaire à celle des Roumains pour comprendre ou apprendre relativement rapidement l'italien ou l'espagnol, les deux langues parlées dans les pays de destination les plus importants. Leur compétence est donc parfois meilleure ou plus riche que celle des Roumains du Maramures ou des Roumains en général, ils sont mieux armés pour l'aventure de la migration dans le contexte actuel de mondialisation. Ce succès de «stratégie de vie » se reflète dans un changement de représentation, d'abord par eux-mêmes, puis par la majorité roumaine qui se voit « obligée » de reconnaître et d'admettre la réussite des Ukrainiens. Cela signifie bien que les identités sociales sont à comprendre et à resituer dans le contexte socio-économique.

Les formes de « débrouille » quotidiennes, qu'elles prennent la forme du tourisme, de la migration ou d'autres stratégies, sont ancrées structurellement dans le passé. Néanmoins, le changement dans les pratiques récentes est, ou plutôt entraîne, un changement de représentation : reprise ou basculement des discours nationalistes, valorisation culturelle par rapport aux nouvelles com-

24. «Le Roumain, quand il veut voler du terrain, il renverse sa clôture et la remet le lendemain là où elle est tombée au sol et voila, il a gagné un mètre... " (témoignage d'un Ukrainien).

25. Appadurai A., Après le colonialisme. Les conséquences culturelles de la globalisation, op. cit. 
pétences nécessaires à cette pluriactivité / multi-stratégie / débrouille économique, etc. L'image et la représentation sont finalement directement liées aux performances et réussites dans les nouvelles formes prises par les plus anciennes stratégies économiques. 